\title{
Congenital Pseudarthrosis of the Tibia with Complex Deformity and Multiple Previous Surgeries Treated by Taylor Spatial Frame at Age of 16 Years
}

\author{
Luft A. Abumunaser, MD, FACHARZT \\ and Mohammed J. Alsayyad, MD, FRCS(C) \\ Department of Orthopedic Surgery, Faculty of Medicine, \\ King Abdulaziz University, Jeddah, Saudi Arabia \\ labumunaser@yahoo.co.uk
}

\begin{abstract}
Congenital pseudarthrosis of the tibia remains one of the most difficult conditions in pediatric orthopedic surgery. The numerous treatment options reflect this difficulty. The aim of successful treatment is to achieve union, length and deformity correction. This reports a 16-years-old male patient with congenital pseudarthrosis of the tibia diagnosed at age of two years, who previously underwent 14 different operations. The patient was referred to King Abdulaziz University Hospital, where he was treated with Taylor Spatial Frame, excision of pseudarthrosis, autogenus bone grafts and Demineralized Bone Matrix was performed, and union was achieved. Stability and deformity correction permitted by Taylor Spatial Frame, in addition to stimulation of bone healing through proper grafting provided a successful option to treat this complex condition.
\end{abstract}

Keywords: Congenital pseudarthrosis of the tibia, Taylor spatial frame, Demineralized bone matrix, Bone graft.

\section{Introduction}

Congenital Pseudarthrosis of tibia (CPT) is one of the most challenging problems in pediatric orthopedics ${ }^{[1]}$. It is an uncommon entity with a reported incidence of $1: 140,000$ to $1: 250,000$ neonates. Bilateral

\footnotetext{
Correspondence \& reprint request to: $\quad$ Dr. Luft A. Abumunaser P.O. Box 80215, Jeddah 21589, Saudi Arabia
} Accepted for publication: 30 December 2009. Received: 26 October 2009. 
occurrence is rare. Usually the disease becomes evident within the first two years of life, but may be undetected till the age of 12 years $^{[2,3]}$. In $40-80 \%$ of patients, it is associated with neurofibromatosis Type 1 (NF1) which generally does not influence the final outcome ${ }^{[4]}$. Approximately $10 \%$ of patients with NF-1 develop pseudarthrosis of tibia $^{[3]}$. The natural history is persistent instability and progressive deformity $^{[5]}$. The condition exhibits a wide range of severity, and the response to the treatment is unpredictable ${ }^{[1]}$. The classification of CPT includes that of Anderson et al. ${ }^{[6]}$, which differentiates the morphology of pseudarthrosis as dysplastic, cystic or sclerotic ${ }^{[6]}$. Crawford et al. ${ }^{[7]}$, described four types of CPT, all have in common an anterolateral bowing of the affected tibia. A limitation of all classifications is the change of the disease morphology caused by children's growth. However, determining the type of the disease at initial imaging is the most important for the prognosis ${ }^{[2]}$. Paley et al. ${ }^{[12]}$, classified CPT into three types, where type I is characterized by atrophic, narrow bone ends, mobile and with no previous surgery. In Type II, however, there is atrophic, narrow bone ends, also mobile, but with previous surgery. Type III have hypertrophic, wide bone ends, stiff, and with or without previous surgery ${ }^{[8]}$. Treatment options have varied greatly and have included both operative and non-operative approaches. Although, no single method has proven ideal, the highest rate of union have been reported with surgery. The surgical procedures mostly used are intramedullary nail (IMN) with bone graft, vascularized fibular graft (VFG) and Ilizarov circular external fixator (CEF) technique ${ }^{[1]}$. Bone morphogenetic proteins (BMPs) have been used successfully in adult patients for treating tibial non-union and spinal fusion. BMP's also facilitates rapid bone regeneration in CPT cases and it is suggested that in addition to standard surgical procedures, local application of bone morphogenetic protein-7 (BMP-7) could safely stimulate bone union ${ }^{[3-9]}$.

In this report, it describes a neglected case of CPT which failed a large number of surgical interventions plus developed severe deformity and shortening; had this deformity corrected achieving union with the six axis deformity correction capability of the ring fixator, the Taylor Spatial Frame (TSF) using the osteoinductive and conductive capabilities of Demineralized Bone Matrix (DBM). 


\section{Case Report}

A sixteen-year-old adolescent was diagnosed at age of one and halfyear with CPT. Fourteen-surgical procedures in attempt to achieve union were performed, including two attempts of open reduction and plating of the tibia with bone grafting. Followed by failure and removal of plates and screw; multiple trials of separate bone grafting procedures with frequent cast changes were done, and finally trial of intramedullary nail with grafting which failed and the hardware was then removed. Due to severe deformity, shortening, pain and stiff ankle with valgus deformity, the patient was referred to the authors. Examination revealed clear stigmata of neurofibromatosis, with severe angulations of the tibia apex anterior $90^{\circ}$ and medial $70^{\circ}$ with leg length discrepancy (LLD) of $17 \mathrm{~cm}$ (Fig. 1A, B, C). Radiographs showed atrophic nonunion of distal tibia with tapered sclerotic ends and obliterated medullary canal, in addition to the above mentioned deformity (Fig. 2A, B). The above description indicates that our case is Type II according to Paley's classification. The decision was to deal with this patient in two stages; first to achieve union with plans to correct residual leg length discrepancy at later stage, as per the patient's request.

A

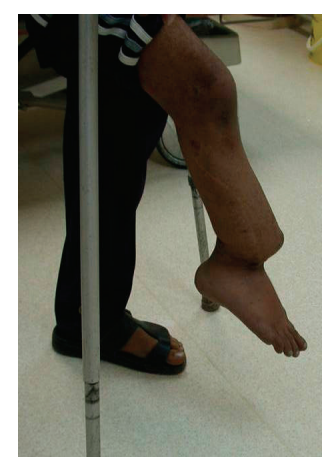

B

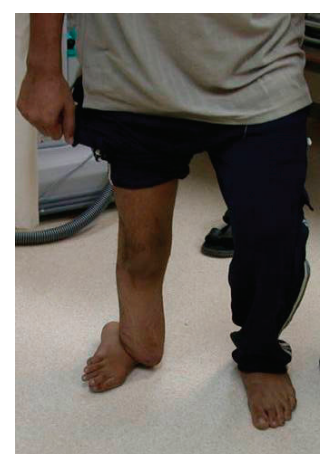

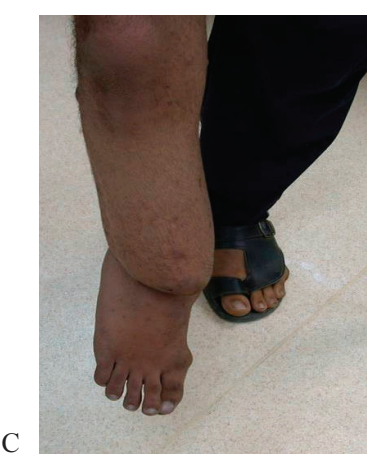

Fig. 1. Preoperative pictures of our patient with CPT demonstrate the obvious deformity. (A) Lateral view. (B) Anterior with weight bearing and (C) Anterior without weight bearing .

The patient was taken to operating room where standard ring first TSF technique was applied proximally and distally on the tibial segments[10]. Then an $8 \mathrm{~cm}$ long incision was made at non-union site, where excision of pseudarthrosis was done. It was ensured that all dysplastic bone has been removed from both sides of the pseudarthrosis site until healthy bleeding bone, though keeping the resection to the 
minimum to avoid unnecessary bone loss. Removal of thickened fibrous tissue of previous surgeries was also performed. However, the deformity was not corrected acutely because of the soft tissue contracture. The proximal fixation included four-half pins of $6 \mathrm{~mm}$ in diameter (hydroxyapatite coated). Distally it was utilized $1.8 \mathrm{~mm}$ Ilizarov wires X 5 (Smith \& Nephew, Memphis, TN USA) holding the short distal fragment. Grafting with periosteum from the iliac crest was performed as follows; periosteum was harvested from the inner table of the iliac crest and was meshed like the split thickness skin graft, and was applied on top of the cleaned pseudarthrosis site. Iliac crest bone graft and $10 \mathrm{cc}$ of DBM (Grafton, Osteotech, Inc., Eatontown, NJ, USA) were performed, then closure in layers (Fig. 3A, B).
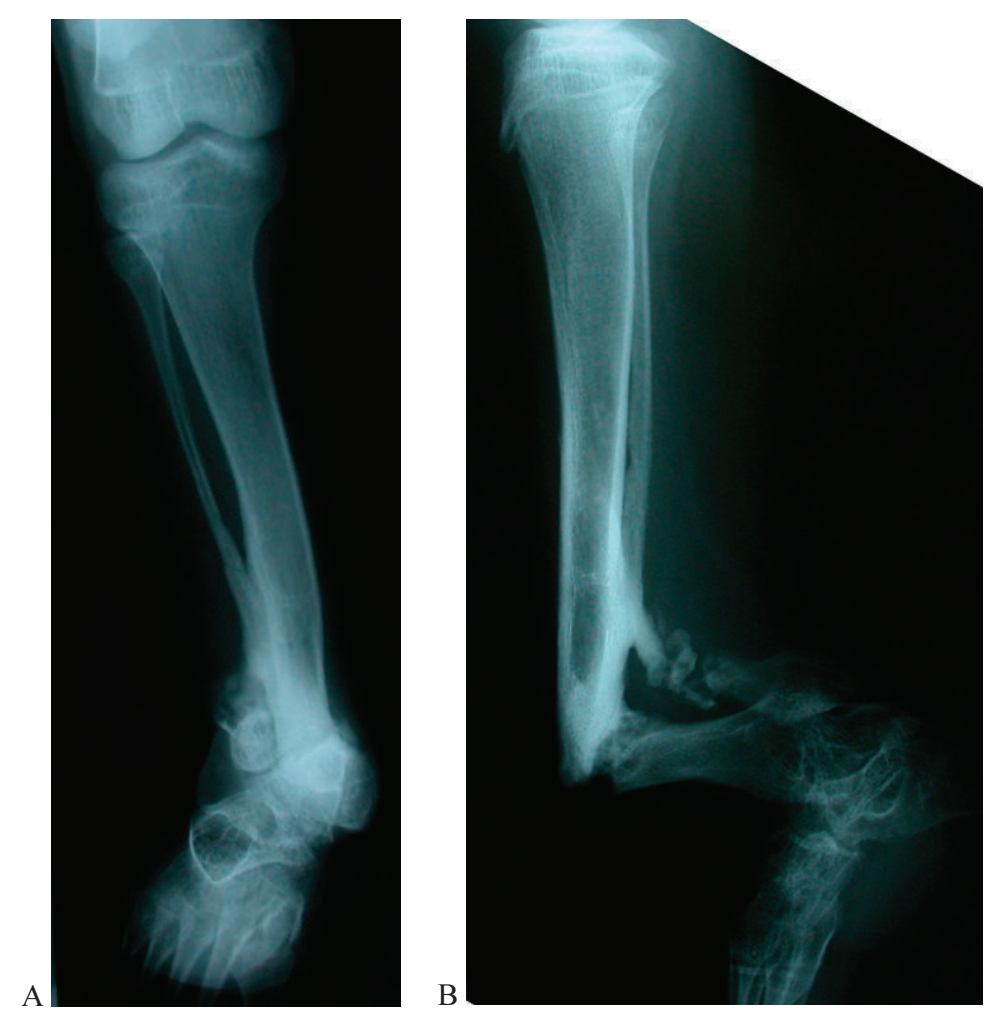

Fig. 2. Preoperative $\mathrm{X}$-ray is showing the anteromedial angular deformity of Rt tibia. (A) Anteroposterior. (B) Lateral view. 

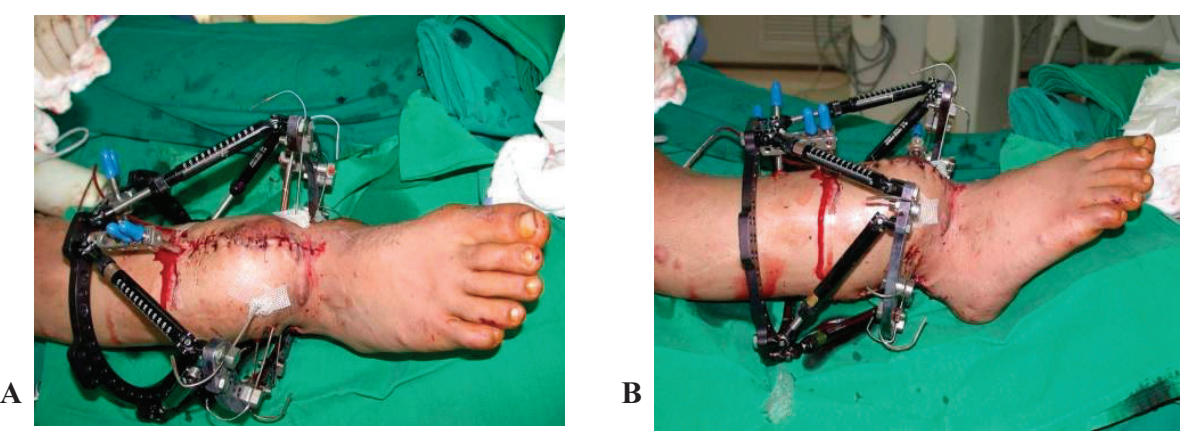

Fig. 3. Immediate post-operative view with TSF: (A) Anterior, (B) Lateral.
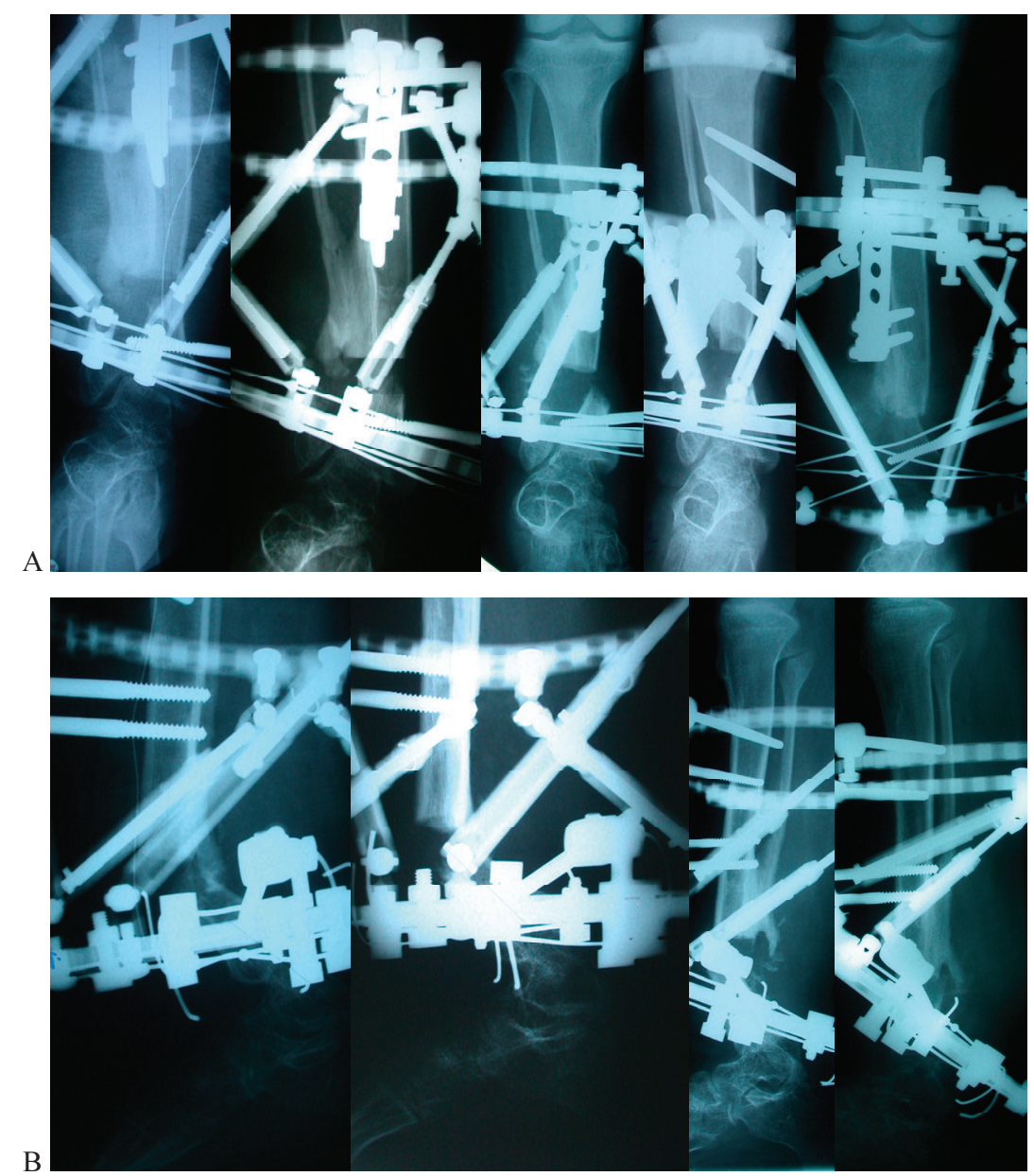

Fig. 4. Gradual correction of the deformity with TSF: (A) Anteroposterior, (B) Lateral view. 
Postoperatively gradual correction of deformity was done utilizing TSF online software program (Smith \& Nephew, Memphis, TN USA) (Figs. 4A, B). Full correction was achieved within 74 days. The patient was mobilized with full weight bearing as tolerated. Union was reached after six months of treatment. This was followed by removal of the frame one month later, after frame dynamization (Figs. 5A, B). The patient continued to utilize braces for four months postoperatively, with $11 \mathrm{~cm}$ leg length discrepancy remaining (Figs. 6A, B, C) and was able to walk for the first time in his life without crutches utilizing a shoe raise of $7 \mathrm{~cm}$. The patient has been followed for 4-years now, and currently is undergoing lengthening using a second TSF. The patient had an excellent range of motion after surgery of $10^{\circ}$ planter-flexion and $40^{\circ}$ dorsi-flexion, and the range remained unchanged with no further improvement in plantar flexion during the follow-up period.

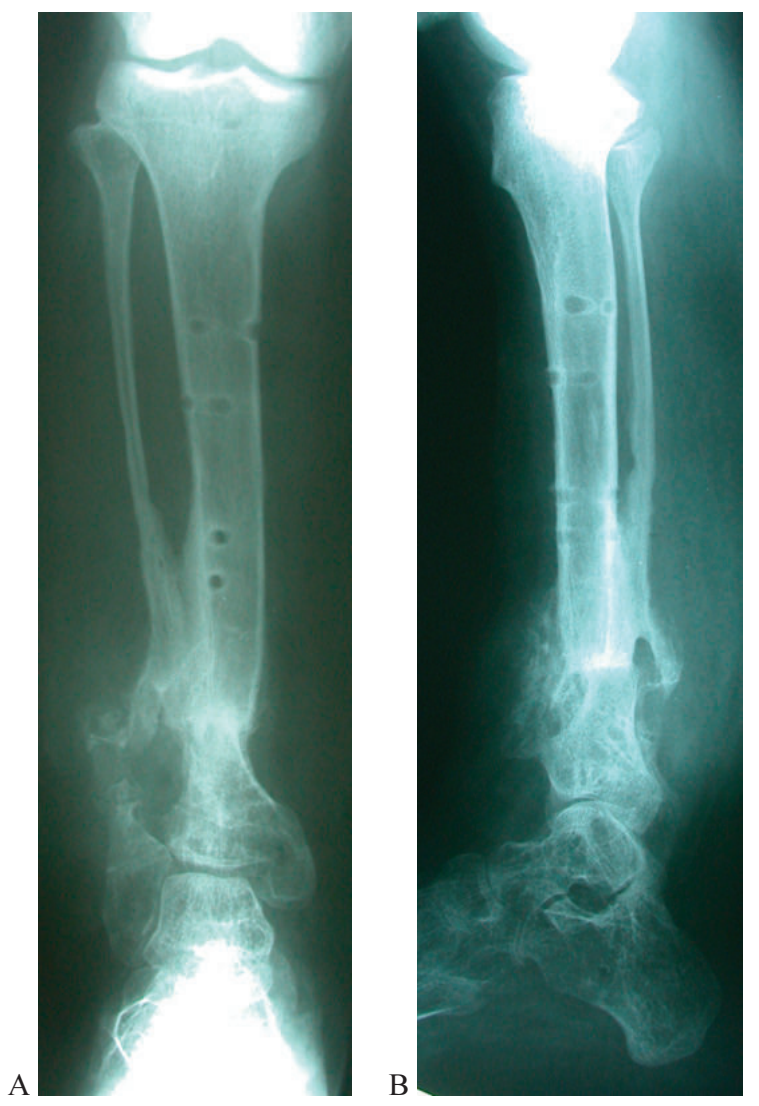

Fig. 5. Solid union and deformity correction: (A) Anteroposterior, (B) Lateral view. 

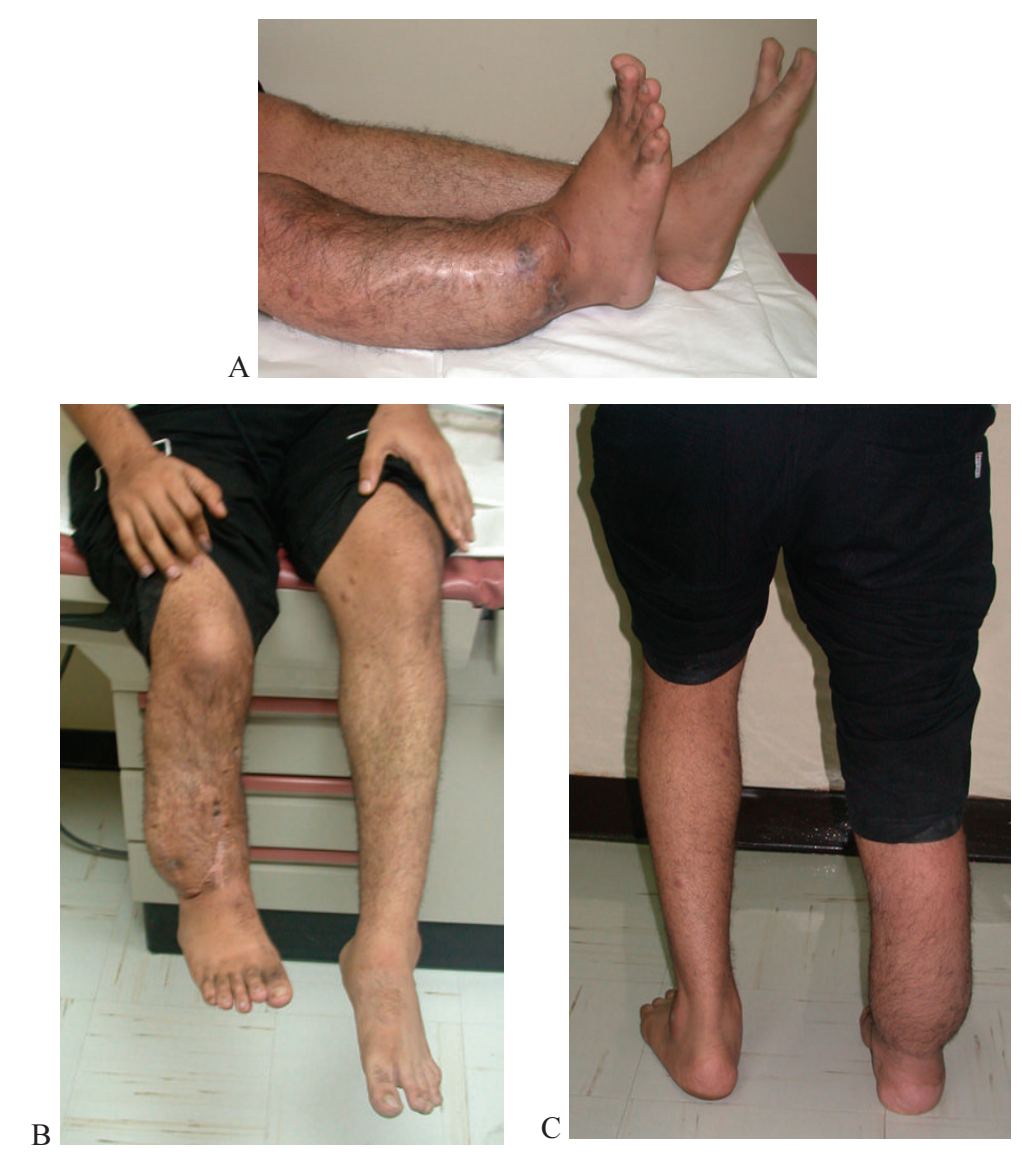

Fig. 6. Full correction of the deformity: (A) Lateral, (B) Anterior view. (C) From posterior, standing with FWB.

\section{Discussion}

Congenital pseudarthrosis of the tibia represents a great challenge to treat in a pediatric orthopedic practice. Treatment measures and in literatures addressing this condition are numerous, reflecting the difficulty in management. The aim of successful treatment is to achieve union, length and normal axis of the affected leg. This success could be possible surgically by achieving the following principles: Correcting the axis of the limb; resection of pseudarthrosis and opening of the medullary canal; lengthening of tibia with proximal osteotomy and concurrent compression of the fracture site ${ }^{[11]}$. The age is an important factor with regard to bone consolidation. According to European Pediatric 
Orthopedic Society (EPOS) multicenter study a clear correlation was shown between age at surgery and final outcome. Therefore, it has been proposed that surgery should not be performed on patients younger than the age of 3 years and recommended to be postponed until the age of 5 years $^{[4]}$. Our patient did have multiple surgeries at a very young age without much success.

The surgical technique of vascularized bone grafting (VBG), intramedullary stabilization with allograft, circular external fixation have been reported to be relatively successful in the treatment of $\mathrm{CPT}^{[4]}$. The superiority of any specific procedure is difficult to determine as that depends on different factors, e.g., type of CPT, the effect of previous operation, age at time of surgery, the need for follow-up until skeletal maturity and definition of what is a successful outcome. All these factors make it difficult to compare the outcome of the different procedures ${ }^{[1]}$. In series of VFG for treatment of CPT, bone consolidation was reported to be obtained in about $94 \%$ of cases, nevertheless complications of refracture, non-union, tibial angular deformities may occur, as well as severe ankle valgus deformity with ankle pain ${ }^{[1-4]}$.

Numerous authors have reported on the use of Ilizarov circular external fixator in the management of $\mathrm{CPT}^{[1]}$. It is a comprehensive approach to all aspects of CPT including resection of pseudarthrosis, deformity correction, shortening the defect, improving joint function and weight bearing. However, its disadvantage is relative complexity, duration of treatment, pin tract infection, refracture and ankle valgus. Paley et al. ${ }^{[12]}$ reported on 16 patients with union rate of $94 \%$ with one treatment and $100 \%$ with two treatments.

Grill et al. in a multicentre study analyzed the different therapeutic methods used by the EPOS ${ }^{[13]}$. The treatment data of 340 patients who underwent 1287 procedures for CPT were analyzed. The therapeutic modalities, which were reviewed, included the McFarland bypass graft, plating, rodding, and grafting, the Ilizarov fixator as well as conservative measures. The findings of that study demonstrated that plating and rodding seemed to afford inadequate stability as to allow the pseudarthrosis to heal. Those surgeons who used that kind of fixation resected too little of the pseudarthrotic bone in an attempt to avoid shortening. The results of that study showed that the Ilizarov technique was the method of choice in the treatment of CPT. In addition to success 
in correction of the other deformities, this method achieved the highest rate of union of $75.5 \%$. The Ilizarov technique is useful in many cases of CPT in which union failed to occur in spite of many previous surgeries. The use of this method does not preclude the use of other procedures, but the Ilizarov method takes considerable time and effort to obtain good results $^{[13]}$.

There have been previous reports in the literature of successful outcomes after the use of TSF in the correction of pediatric lower limb deformities, and in the acute sitting ${ }^{[14-16]}$. Sluga et al. reported the use of TSF in a series of 5 children with shortened and deformed lower extremities, of which 4 due to fracture, osteomyelitis, and a congenitally short femur, and 1 patient had a pseudoarthrosis of the tibia. The mean elongation achieved was $5.9 \mathrm{~cm}$ and a mean valgus correction of $10.6^{\circ}$ and a mean lateral translation of $12.6 \mathrm{~mm}$. In all patients, bones fused without major complications or residual deformity ${ }^{[15]}$. Eidelman et al. reported their experience with the use of the TSF in pediatric deformities, achieving an anatomical correction in 30 of the 31 children treated ${ }^{[16]}$. Al-Sayyad recently described his encouraging results in the use of TSF in treating 10 unstable tibial fractures, including 5 open fractures, showing a mean fracture healing time of 18 weeks ${ }^{[17]}$. Recently, the TSF has been compared with the Ilizarov external fixator for tibial lengthening. Although no difference was found in the mean lengthening time or complication rate, the authors noted that multiplanar deformity correction was easier to perform with $\operatorname{TSF}^{[18]}$.

Naqui et al. ${ }^{[19]}$ treated 3 neurofibromatosis pseudoarthrosis. One is a teenage patient with a history of psychiatric problems, who had a stacked frame for pseudarthrosis of the tibia, refused to weight bear and demanded the frame to be removed before full regenerate formation. At this time, there was no residual deformity. He eventually returned and requested further treatment. A second frame was applied 14 months later addressing the subsequent fibrous malunion, and residual deformity of 10-degree valgus and $10-\mathrm{mm}$ shortening. The second treatment was without complication; he had no residual deformity and a full range of movement at the knee and ankle ${ }^{[19]}$. The result in this patient was obtained after prolonged use of TSF. While in our patient, the union was achieved after only 6 months and in one surgical procedure. 
This case demonstrated a success of TSF in obtaining easy correction of a severe deformity with improvement of contact area, which in return promotes healing, in addition to permitting excellent stability and ability to an early weight bear. The addition of DBM improves osteoinduction and conduction.

\section{References}

[1] Rose RE, Wright DE. Treatment of congenital pseudarthrosis of the tibia with the Ilizarov technique. Case report and review of the literature. West Indian Med J 2007; 56(3): 294299.

[2] Mahnken AH, Staatz G, Hermanns B, Gunter RW, Weber M. Congenital pseudarthrosis of the tibia in pediatric patients: MR imaging. AJR Am J Roentgenol 2001; 177(5): 1025-1029.

[3] Fabeck L, Ghafil D. Gerroudj M. Bailon R, Delince PH. Bone morphogenetic protein 7 in the treatment of congenital pseudarthrosis of the tibia. J Bone Joint Surg Br 2006; 88(1): 116-118.

[4] Morissy RT. Cogenital pseudarthrosis of the tibia. Factors that affect results. Clin Orthop Relat Res 1982; 166: 21-27.

[5] Sakamoto A, Yoshida T, Uchida Y, Kojima T, Kubota H, Iwamoto Y. Long-term follow-up on the use of vascularized fibular graft for the treatment of congenital pseudarthrosis of the tibia. J Orthop Surg Res 2008; 3: 13.

[6] Anderson KS. Radiological classification of congenital pseudarthrosis of the tibia. Acta Orthop Scand 1973; 44(6): 719-727.

[7] Crawford AH. Neurofibromatosis in children. Acta Orthop Scand Suppl 1986; 218: 160.

[8] El-Rosasy MA, Paley D, Herzenberg JE. Congenital pseudarthrosis of the tibia. In: Rozbruch RS, Ilizarov S, (eds.) Limb Lengthening and Reconstruction Surgery. New York: Informa Healthcare, 2007. Chp 34, 485-493.

[9] Anticevic D, Jelic M, Vukicevic S. Treatment of a congenital pseudarthrosis of the tibia by osteogenic protein-1 (bone morphogenetic protein-7): a case report. J Pediatr Orthop B 2006; 15(3): 220-221.

[10] Binski J. Taylor Spatial Frame in Acute Fracture Care. Techniques in Orthopedics 2002; 17(2): 173-184.

[11] Odeski Y. The surgical management of congenital pseudarthrosis of the tibia. $J$ Bone Joint Surg Br 2002; 84-B (SUPP III): 307-308.

[12] Paley D, Catagni M, Argnani F, Prevot J, Bell D, Armstrong P. Treatment of congenital pseudarthrosis of the tibia using the Ilizarov technique. Clin Orthop Relat Res 1992; 280: 81-93.

[13] Grill F, Bollini G, Dungl P, Fixsen J, Hefti F, Ippolito E, Romanus B, Tudisco C, Wientroub S. Treatment approaches for congenital pseudarthrosis of tibia: results of the EPOS multicenter study. European Paediatric Orthopaedic Society (EPOS). J Pediatr Orthop B 2000; 9(2): 75-89.

[14] Fadel M, Hosny G. The Taylor spatial frame for deformity correction in the lower limbs. Int Orthop 2005; 29(2): 125-129. 
[15] Sluga M, Pfeiffer M, Kotz R, Nehrer S. Lower limb deformities in children: two-stage correction using the Taylor spatial frame. J Pediatr Orthop B 2003; 12(2): 123-128.

[16] Eidelman M, Bialik V, Katzman A. Correction of deformities in children using the Taylor spatial frame. J Pediater Orthop B 2006; 15(6): 387-395.

[17] Al-Sayyad MJ. Taylor spatial frame in the treatment of pediatric and adolescent tibial shaft fractures. J Pediater Orthop 2006; 26(2): 164-170.

[18] Kristiansen LP, Steen H, Reikerås O. No difference in tibial lengthening index by use of Taylor spatial frame or Ilizarov external fixator. Acta Orthop 2006; 77(5): 772-777.

[19] Naqui SZ, Thiryayi W, Foster A, Tselentakis G, Evans M, Day JB. Correction of simple and complex pediatric deformities using the Taylor-Spatial Frame. J Pediatr Orthop 2006; 28(6): 640-647. 


\title{
علاج حالة مفصل كاذب خلقي لعظمة الساق ذي تشوه معقد وبعد عمليات جر احية متعددة، بو اسطة جهاز "Taylor Spatial Frame"
}

\author{
لطف أحمد أبومنصرّ و محمد جلال الصيّاد \\ قسم جر/حة العظام ، كلية الطب ، جامعة الملك عبدالعزيز$$
\text { جلة - المدلكة العربية السعودية }
$$

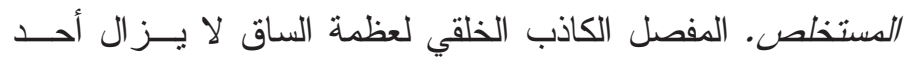

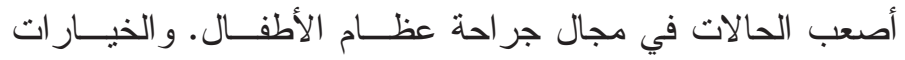

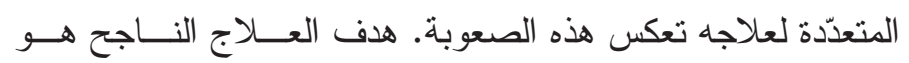

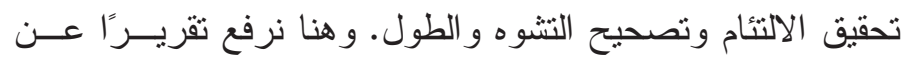

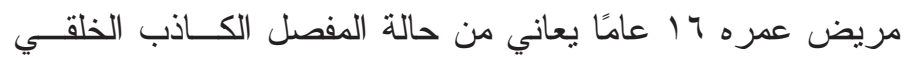 \\ لعظمة الساق، وقد حوّل المريض إلينا وتم علاجه بواسطة جهــاز

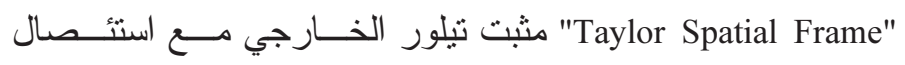 \\ المفصل الكاذب وزر ع نسيج عظمي ذاتي (Autogenus bone graft)

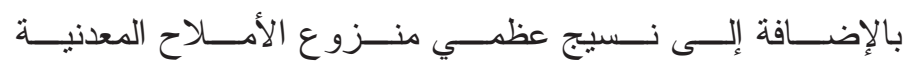 \\ وقد تم تحقيق التئام العظم الثباتيـــة (Demineralized bone matrix) \\ وتصحيح التشوه بواسطة جهاز مثبت تيلور الخارجي، بالإضــافة وفة \\ إلى تتشيط وحث التئام العظم عن طريق الزر اعة العظمية المناسبة \\ الذي يعتبر خيارًا ناجحًا لعلاج هذه الحالة المعقدة.
}

